\title{
Numerical analysis of in vivo platelet consumption data from ITP patients
}

Ted S. Strom ${ }^{1,2}$

\begin{abstract}
Background: Numerical methods have recently allowed quantitative interpretation of in vivo murine platelet consumption data in terms of values for the random destruction rate constant (RD), intrinsic lifespan (LS), and the standard deviation of In LS (SD), as well as the platelet production rate (PR) and age distribution (AD). But application of these methods to data obtained in thrombocytopenic patients is problematic for two reasons. First, such data has in all cases been obtained with radiolabeled platelets, and uptake of the radio-isotope by long lived cells complicates the analysis. Second, inferred values of the platelet production rate (PR) and random destruction rate (RD) are difficult to interpret, since increased RD can occur either as a cause or a consequence of thrombocytopenia.
\end{abstract}

Methods: We used a numerical method to analyze in vivo platelet consumption data from a series of 41 patients with immune thrombocytopenic purpura (ITP). An additional parameter, the fraction of labeled long-lived cells (LL), was evaluated concurrently with RD, LS, and SD. To provide a basis for interpreting these values, we used an iterative interpolation process to predict their response to different pathophysiologic mechanisms. The process also generates predicted effects on the widely used immature platelet fraction (IPF).

Results: Optimal parameter value sets were identified in $76 \%$ (31 of 41) of the data sets. 27 of 31 ITP patients showed no substantial homeostatic increase in platelet production, with the remaining 4 showing both augmented platelet consumption and a compensatory increase in PR. Up to 1/3 of the patients showed the degree of increased RD expected to result from reduced thrombopoiesis only. "Jacknife" resampling yielded CV values of $<0.5$ in over $75 \%$ of the evaluable data sets. Predicted platelet age distributions indicate that interpretation of the IPF and absolute IPF (alPF) is a complex function of platelet count. We found, counter-intuitively, that reduced PR can increase the IPF, and increased RD can reduce the alPF.

Conclusions: Our findings support the feasibility of using numerical analysis to quantitatively interpret in vivo platelet consumption data, to identify likely etiologies of thrombocytopenias, and to assess the utility of IPF measurements in that context.

Keywords: Platelets, Thrombocytopenia, Immune thrombocytopenic purpura, Numerical analysis

\section{Background}

In vivo platelet consumption studies have often been used to quantify the rates of both random and lifespandependent consumption processes, and to evaluate production rate, but their interpretation can be problematic. The aim of the present study is to demonstrate that a numerical analysis method can reliably quantify these

\section{Correspondence: tstrom@uthsc.edu}

'Department of Pathology and Laboratory Medicine, Memphis Veterans Administration Medical Center, 1030 Jefferson Ave, Memphis, TN 38104, USA ${ }^{2}$ Department of Pathology and Laboratory Medicine, University of Tennessee Health Sciences Center, Memphis, TN, USA rates, and thereby identify fundamental pathophysiologic features, in thrombocytopenic patients.

Methods which have been used to interpret such studies include a simple exponential decay model [1], a weighted mean method that applies an empirical mixture of separately optimized linear and exponential decay processes [2], a purely lifespan-dependent model [3], the Mills-Dornhorst equation (which includes but does not solve for a random (exponential) consumption rate constant) $[4,5]$, the widely used multiple hit model (based on a unique consumption mechanism for which 
there is little experimental support) [6, 7], and combined use of the latter two approaches $[8,9]$.

None of these methods allow concurrent modeling of the random (hemostatic and phagocyte-mediated) and lifespan-dependent processes known to result in most in vivo platelet consumption. Numerical analysis models have been designed for that purpose, and their utility for the analysis of murine platelet consumption data has been demonstrated $[10,11]$.

It is not clear, however, whether these methods can be adapted to evaluate existing clinical data, since the latter in all cases involves tracking of radiolabeled platelets and includes contributions from uptake of the radioisotope by other, longer-lived cell types. It is also unclear how to translate the resultant kinetic parameter values into useful conclusions about how individual patients became thrombocytopenic. Here we have modified a previously described numerical model [10] to successfully analyze and interpret published data from a series of patients with immune thrombocytopenic purpura (ITP) [12].

\section{Methods \\ Patients}

Entry criteria for the ITP patients have been described previously [12]. The study, including informed consent procedures, was performed according to the principles outlined in the Declaration of Helsinki of 1975. Briefly, ${ }^{111}$ Indium-labeled autologous platelet consumption data from 41 consecutive adult patients with prednisone non-responsive primary ITP was reviewed. The data was obtained either (A) at the time of diagnosis, or (B) after failure to sustain a platelet count response to prednisone treatment. Diagnostic criteria for all patients included exclusion of other malignant, metabolic, or pharmacologic causes, as well as causes of "secondary" ITP such as hepatitis $\mathrm{C}$ virus (HCV) infection. For those in group (A), rapid consumption of autologous ${ }^{111}$ Indium-labeled platelets (interpreted via the multiple hit model [6]) was an additional diagnostic criterion. For those in group (B), demonstration of antiplatelet antibodies on the platelet surface via indirect immunofluorescence [13] was used for this purpose.

All of the patients in this study underwent splenectomy after failing to respond adequately to prednisone treatment. Patients were deemed to have had a complete response to splenectomy if their platelet count persistently exceeded $100 \times 10^{9} / \mathrm{L}$ thereafter, with no significant bleeding episodes. They were considered "non-responders" if their subsequent platelet count did not exceed either $30 \times$ $10^{9} / \mathrm{L}$, or twice their baseline count, or if they had persistent significant bleeding episodes.

\section{Platelet kinetics studies}

These have been described in detail previously [12]. Briefly, platelet rich plasma was prepared by differential centrifugation, and platelets prepared by a subsequent high speed centrifugation were labeled with ${ }^{111}$ Indium oxine by standard methods. Peripheral blood specimens obtained at $30 \mathrm{~min}$ after injection were considered "baseline" measurements (for patient 30, a $1.5 \mathrm{~h}$ time point was used), and all subsequent measurements were normalized to these for each patient. Equilibration with a pool of splenic platelets is thought to be complete well within this initial time frame [14]. All post-injection specimens for each patient were evaluated in a gamma counter at the same time to eliminate decay-related effects on recovery.

\section{Numerical analysis}

Data analysis was performed on desktop computers using Microsoft Excel. Baseline (initial) parameter ranges searched were RD 0-19 (resolution $1 \%$ ) \%/h, LS 0$15.2 \%$ (resolution $0.8 \%$ ), and SD 20-267 h (resolution $13 \mathrm{~h}$ ). LL and RD ranges and resolutions were then empirically optimized to the values shown in Table 1 . The equilibration metric was calculated as the net platelet count produced by the model at the midpoint of the equilibration phase divided by the net platelet count at the end of the equilibration phase, as described previously [10]. All searches achieved an equilibration metric value of $>0.997$ (1000 interval equilibration phase, $0.5 \mathrm{~h}$ per interval). Searches of resampled data sets were performed over the same parameter ranges shown in Table 1. For cases in which all resampled data sets yielded the same parameter values as the complete data set at resolution " $R$ ", the upper limit of the standard deviation was estimated by the value obtained had one resampled data set yielded a parameter value one " $R$ " range removed from that of the complete data set.

\section{Results and discussion}

\section{Modeling in vivo platelet turnover}

The numerical model used here [10] posits that an in vivo platelet population can be visualized in a spreadsheet as a series of small platelet cohort concentrations. The cohorts are assumed to be produced at a constant rate $(\mathrm{PR}, \mathrm{K} / \mathrm{ul} / \mathrm{h})$ in short sequential time periods, and individually consumed, by both random and lifespandependent processes, at the end of each such time period. The consumption curve for individual cohorts is determined by a random destruction rate constant (RD, $\% / \mathrm{h}$ ), by the lognormally distributed cohort lifespan (LS, hr), and by the standard deviation of ln LS (SD). Population platelet consumption curves are generated by summing the cohort values at sequential time points. Optimal theoretical consumption curves generated by a 
Table 1 Patient characteristics, search parameter ranges, and optimal parameter values

\begin{tabular}{|c|c|c|c|c|c|c|c|c|c|c|c|c|c|}
\hline \multicolumn{2}{|c|}{ Patient characteristics } & \multicolumn{3}{|c|}{$\underline{\text { Search parameter ranges }}$} & \multicolumn{9}{|c|}{ Optimal parameter values and residuals (SS/n) } \\
\hline Patient & $\begin{array}{l}\text { Platelets (X } \\
\text { 10e9/L) }\end{array}$ & $\begin{array}{l}\mathrm{n} \text { (data } \\
\text { points) }\end{array}$ & $\mathrm{RD}(\% / \mathrm{h})$ & LL (\%) & $\begin{array}{l}\mathrm{RD} \\
(\% / \mathrm{h})\end{array}$ & CV & LS (hr) & $\mathrm{CV}$ & $\mathrm{LL}(\%)$ & CV & $\begin{array}{l}\text { SD (of } \\
\text { In LS) }\end{array}$ & $\mathrm{PR}(\mathrm{K} / \mathrm{ul} / \mathrm{h})$ & $\mathrm{SS} / \mathrm{n}$ \\
\hline 1 & 30 & 6 & $0-4.75$ & $0-15.2$ & 2.5 & 0.08 & 189 & 0.39 & 6.4 & 0.44 & 0.2 & 0.77 & 0.43 \\
\hline 2 & 119 & 9 & $0-4.75$ & $0-15.2$ & 0.75 & 0.39 & 228 & 0.2 & 12.8 & 0.3 & 0.2 & 0.89 & $6 . .92$ \\
\hline 3 & 165 & 9 & $0-4.75$ & $0-15.2$ & 2 & 0.15 & 176 & 0.2 & 8.8 & 0.11 & 0.3 & 3.55 & 1.81 \\
\hline 4 & 24 & 9 & $4.5-9.25$ & $0-15.2$ & 6.75 & 0.13 & 267 & $n a^{*}$ & 4.8 & 0.31 & 0.2 & 1.62 & 8.26 \\
\hline 5 & 80 & 9 & $3.5-8.25$ & $0-15.2$ & 5.5 & 0.06 & 215 & $n a^{*}$ & 4.8 & 0.23 & 0.2 & 4.4 & 2.61 \\
\hline 7 & 41 & 9 & $1.0-5.75$ & $0-15.2$ & 3.5 & 0.06 & 176 & 0.51 & 6.4 & $<0.13^{* *}$ & 0.1 & 1.44 & 12 \\
\hline 8 & 58 & 8 & $0-4.75$ & $15.2-30.4$ & 2 & 0.43 & 202 & 0.36 & 23.2 & 0.44 & 0.1 & 1.64 & 42.5 \\
\hline 11 & 10 & 5 & $9.5-14.75$ & $0-15.2$ & 12.5 & 0.02 & 267 & $n a^{*}$ & 7.2 & 1.81 & 0.2 & 1.25 & 19.3 \\
\hline 13 & 44 & 6 & $0-4.75$ & $0-15.2$ & 1.5 & 0.28 & 189 & 0.39 & 9.6 & 0.63 & 0.1 & 0.71 & 15.8 \\
\hline 14 & 17 & 5 & $9.5-15.25$ & $0-15.2$ & 12.25 & 0.11 & 72 & 2.94 & 0 & $\left(s d<0.06^{* *}\right)$ & 0.3 & 2.08 & 7.02 \\
\hline 17 & 8 & 9 & $0-4.75$ & $0-15.2$ & 1.75 & 0.2 & 215 & 0.56 & 10.4 & 1.05 & 0.1 & 0.14 & 36.3 \\
\hline 18 & 88 & 7 & $0-4.75$ & $0-15.2$ & 2 & 0.2 & 150 & 0.77 & 8 & 0.34 & 0.2 & 1.92 & 3.3 \\
\hline 20 & 45 & 9 & $2.5-7.25$ & $0-15.2$ & 5.25 & 0.11 & 228 & 0.4 & 6.4 & 0.42 & 0.2 & 2.36 & 22.1 \\
\hline 21 & 143 & 9 & $0-4.75$ & $8.8-24.0$ & 0 & $\left(\mathrm{sd}<0.22^{* *}\right)$ & 124 & $<0.12^{* *}$ & 11.2 & 0.16 & 0.2 & 1.34 & 5.82 \\
\hline 22 & 85 & 8 & $0-4.75$ & $0-15.2$ & 1.25 & 0.32 & 111 & 0.14 & 5.6 & 0.43 & 0.3 & 1.61 & 1.49 \\
\hline 23 & 119 & 9 & $0-4.75$ & $15.2-30.4$ & 0 & $(\mathrm{sd}=0.22)$ & 124 & 0.09 & 22.4 & 0.07 & 0.3 & 1.2 & 4.03 \\
\hline 24 & 20 & 6 & $0-4.75$ & $0-15.2$ & 2.5 & 0.8 & 33 & 0.66 & 1.6 & 0.42 & 0.3 & 1.04 & 21.4 \\
\hline 25 & 39 & 9 & $2.0-6.75$ & $0-15.2$ & 4.5 & 0.09 & 228 & 0.75 & 4.8 & 0.2 & 0.2 & 1.76 & 8.2 \\
\hline 27 & 2 & 9 & $5.5-10.25$ & $0-15.2$ & 8.25 & 0.06 & 176 & 0.73 & 11.2 & 0.18 & 0.1 & 0.17 & 4.77 \\
\hline 28 & 22 & 9 & $0-4.75$ & $0-15.2$ & 2.25 & 0.2 & 254 & 0.35 & 13.6 & 0.25 & 0.1 & 0.5 & 24.4 \\
\hline 29 & 34 & 9 & $2.0-6.75$ & $0-15.2$ & 4.5 & 0.23 & 189 & 0.85 & 6.4 & 0.4 & 0.1 & 1.53 & 18.1 \\
\hline 30 & 13 & 6 & $0-4.75$ & $0-15.2$ & 1.75 & 0.24 & 137 & 0.25 & 6.4 & 1.05 & 0.2 & 0.26 & 6.28 \\
\hline 32 & 36 & 5 & $3.0-7.75$ & $0-15.2$ & 7.25 & 0.04 & 72 & 0.53 & 2.4 & 0.27 & 0.1 & 2.63 & 0.38 \\
\hline 33 & 238 & 7 & $0-4.75$ & $13.6-28.8$ & 0.25 & 2.56 & 163 & 0.47 & 20.8 & 0.39 & 0.2 & 2.33 & 10.2 \\
\hline 34 & 77 & 6 & $2.0-6.75$ & $16.8-32.0$ & 4.5 & 0.27 & 124 & 0.35 & 24 & 0.23 & 0.1 & 3.49 & 5.51 \\
\hline 35 & 37 & 9 & $0.5-5.25$ & $0-15.2$ & 3 & 0.48 & 137 & 1.02 & 5.6 & 0.36 & 0.3 & 1.17 & 13.4 \\
\hline 36 & 32 & 6 & $4.5-9.25$ & $0-15.2$ & 5.75 & 0.15 & 111 & 0.58 & 6.4 & 0.95 & 0.2 & 1.85 & 5.88 \\
\hline 37 & 102 & 6 & $0-4.75$ & $11.2-26.4$ & 3 & 0.58 & 228 & 0.31 & 17.6 & 0.43 & 0.1 & 3.07 & 49.5 \\
\hline 38 & 170 & 7 & $0-4.75$ & $17.6-32.8$ & 0.25 & 1.2 & 150 & 0.07 & 24.8 & 0.05 & 0.1 & 1.45 & 2.81 \\
\hline 39 & 21 & 6 & $3.0-7.75$ & $0-15.2$ & 5.25 & 0.35 & 111 & 1.27 & 12.8 & 0.39 & 0.2 & 1.12 & 27.3 \\
\hline 40 & 43 & 6 & $2.5-7.25$ & $0-15.2$ & 5 & 0.35 & 124 & $n a^{*}$ & 14.4 & 0.41 & 0.2 & 2.16 & 15.8 \\
\hline Normalized & 191 & 23 & $0.05-1.0$ & na & 0.5 & & 140 & & na & & 0.2 & 2.12 & 57.3 \\
\hline
\end{tabular}

Platelet counts were obtained at the time of the study. Patients 1,2, and 3 showed a major subsequent response to splenectomy (see text). Resolution is equal to $5 \%$ of the search ranges shown. The "normalized" data set is pooled data from the three patients $(3,33$, and 38$)$ whose platelet counts transiently exceeded $150 \mathrm{~K} / \mathrm{ul}$ in response to prednisone. CV values were obtained by "jackknife" resampling (see text). "na*" denotes cases for which one or more of the resampled or complete data sets yielded LS values at the high end of the search range. Values marked by ** were for cases in which all of the resampled data sets yielded the same optimal parameter value (see Methods)

large range of possible parameter values are identified via quantitative comparison to each data set (summed squared residual values, or SS).

For the 41 patient studies analyzed here, each patient's platelet consumption data was normalized to the first (baseline) measurement of circulating ${ }^{111} \mathrm{In}$. Visual inspection of the data strongly suggests that many of the labeled platelet preparations contained long lived species, as others have described in similar studies $[15,16]$. This is evident in the plateau phase seen at late times in the consumption data (for example, patients 35 and 27, Fig. 4). To take this into account, we evaluated a fourth parameter: the fraction of the labeled cells/platelets consisting of long lived (species (LL, \%). This parameter simply "shrinks" the scope of the analysis to the consumption of platelets from $100 \%$ of the time zero value to an optimizable minimum percentage 
(LL). For modeling purposes, lifespans of these long lived species are assumed to be infinite.

\section{Optimal parameter value search process}

Optimal parameter value searches were performed as shown schematically in Fig. 1. For a given data set, SS values are determined for each possible set of parameter values in a four-dimensional parameter space defined by RD, LS, SD, and LL. The core component of the search is an evaluation of SS for each point in a $20 \times 20$ plane of possible LS and LL values at fixed values of RD and SS. The resultant minimum "planar" SS values are visually identifiable (see examples in Fig. 2). This process is repeated over a range of $20 \mathrm{RD}$ values, yielding in most cases single "volume" SS minima as shown in Fig. 3a. Finally, the entire process is repeated at a series of SD values, and the resultant volume minima are compared in order to identify a "global" minimum SS value and its associated parameter values. Distinguishable alternative volume minima showing SS values greater than those of the global minima were also seen in some data sets (see below). Searches were performed for only three SD values, as this generated a plausible range of distribution widths for the resultant lifespan-dependent consumption rates (see examples in Fig. 3b) while significantly reducing computation time. Examples of the consumption curves generated by the optimal parameter values are shown in Fig. 4.

\section{Data quality evaluation and optimal parameter value search results}

This process is outlined in Fig. 5. Of the 41 originally reported ITP patient data sets, one was excluded due to lack of initial time point data. One patient demonstrated an initial platelet clearance rate of $46 \%$ in the first $1.5 \mathrm{~h}$ of the study ( $>4$ standard deviations faster than the mean). Because this value suggests the type of platelet activation during labeling/processing that we have on occasion seen in murine platelet clearance studies (TS, unpublished), this data was also excluded. For the remainder, quality of parameter value optimization was evaluated in terms of the ratio of SS to $\mathrm{n}$ (the number of data points per patient data set), where $\mathrm{n}$ ranged from 5 to 9 (Table 1). One case (patient 31) with an $\mathrm{SS} / \mathrm{n}$ value of 143 (over four standard deviations from the mean value of 18.5) was then excluded. No other SS/n values fell beyond two sd from the mean.

A single "global" minimum SS value, with its associated (optimal) parameter values, was identified in 24 of the 39 evaluable data sets. The optimal consumption curves show a large amount of inter-patient variation, as the examples in Fig. 4a demonstrate. Of those showing more than one minimum, convincing global minima were identified in four data sets on the basis of goodness-of-fit. Specifically, the global minima in these cases showed SS values which were less than $50 \%$ of those defining the alternative (local) minima. Three data sets showed global minima for which comparison of absolute vs. squared residuals provided additional support for their significance (see Additional file 1). Seven data sets, however, showed local minima that could not be distinguished from the global minima on these bases. In sum, we were able to identify convincing global minima in 31 of the 39 evaluable data sets (79 \%) shown in Table 1.

Data quality was further evaluated by performing "jackknife" resampling studies on each of the patient data sets in Table 1 [17]. Optimal parameter values were obtained for each of the $n$ subsets for each data set via the same process

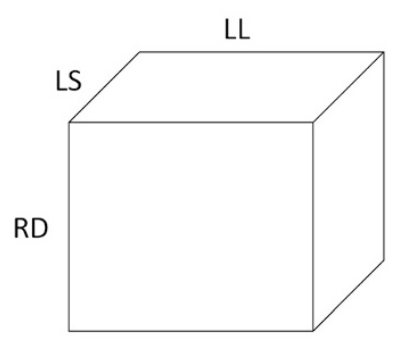

Parameter space for $R D, L S, L L$ $(20 \times 20 \times 20)$ at $S D_{1}$

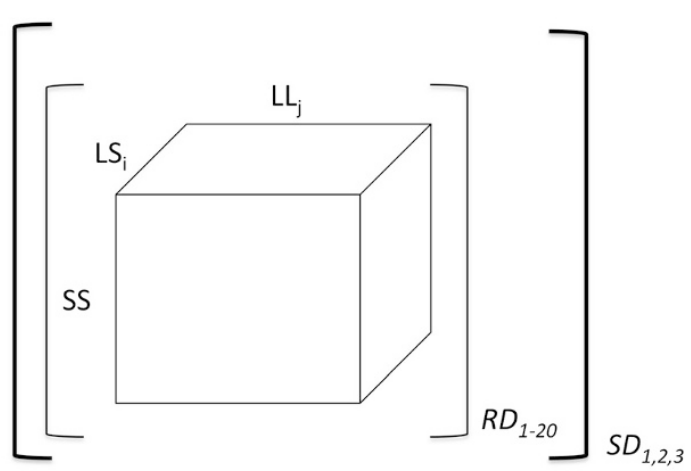

Search sequence for parameter space at three $S D$ values

Fig. 1 Optimal parameter search schematic. Left: parameter space evaluated for each data set. Right: Schematic of search sequence used. For each data set, SS values were calculated for each point in each RD-defined plane in parameter space. Minimal SS values were identified for each plane; each plane was evaluated at 20 RD values; and each RD value was evaluated at 3 SD values 


\section{Patient 35: SS value minima in sequential RD-defined planes}

$R D, \% / h r:$

2.75

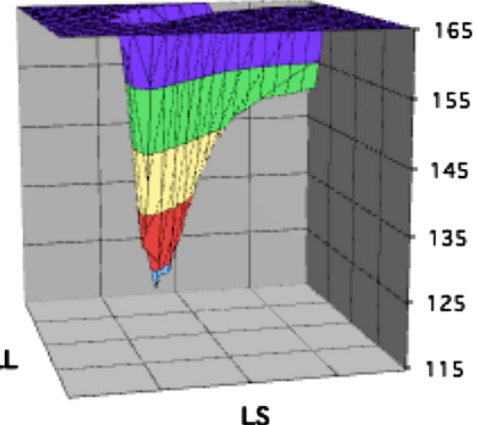

3.0

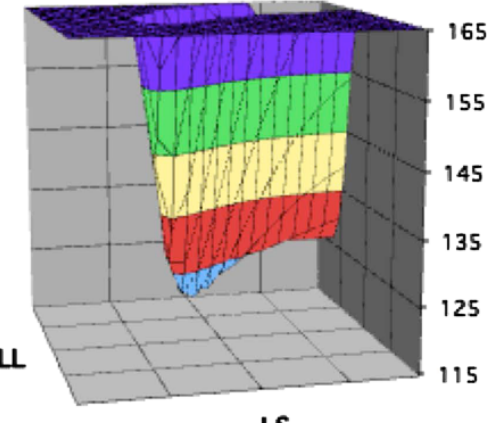

3.25

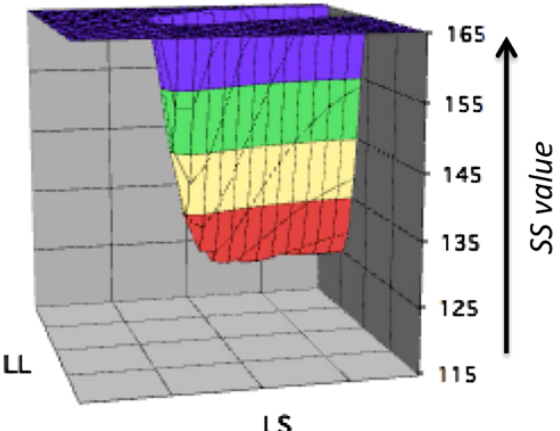

Fig. 2 Identifying optimal parameter values. The graphs demonstrate three 'planar' SS value minima, of which the minimum in the plane defined by $\mathrm{RD}=3.0 \% / \mathrm{h}$ is the 'volume' minimum at this SD value (0.3). The parameter values associated with this SS minimum yield the consumption curve for this patient shown in Fig. 4. Horizontal axis scales are as follows: For LL, 0 to $15.2 \%$, resolution $0.8 \%$. For LS, 20 to 267 h, resolution 13 h. See Table 1 for additional data

A) SS value minima in sequential RD-defined plates
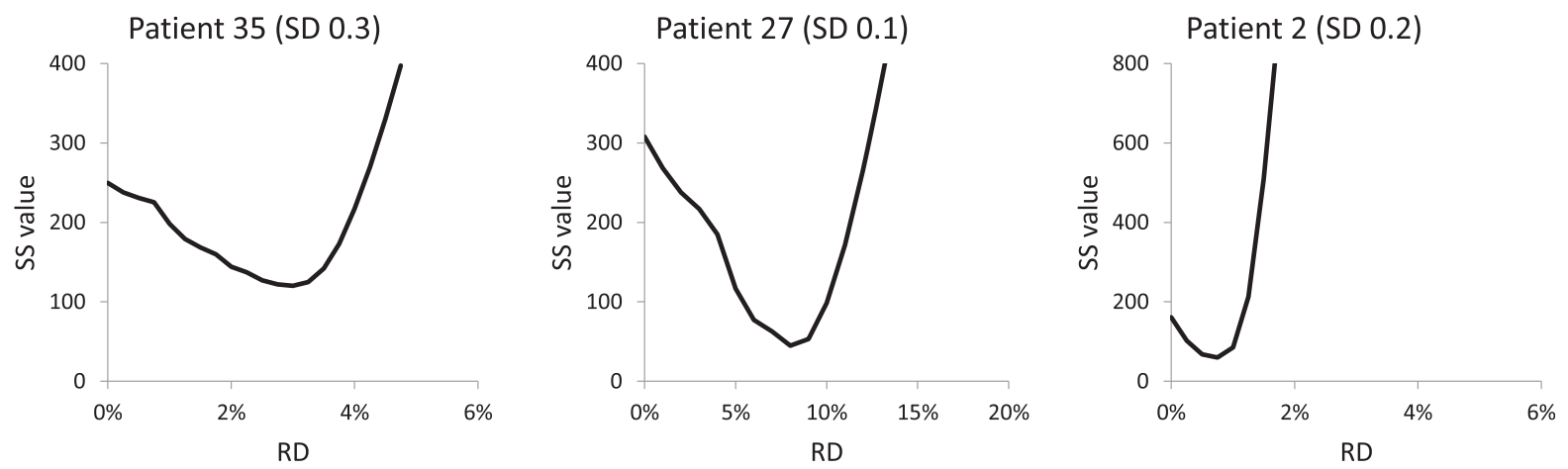

B) Optimal fractional lifespan-dependent platelet consumption rates
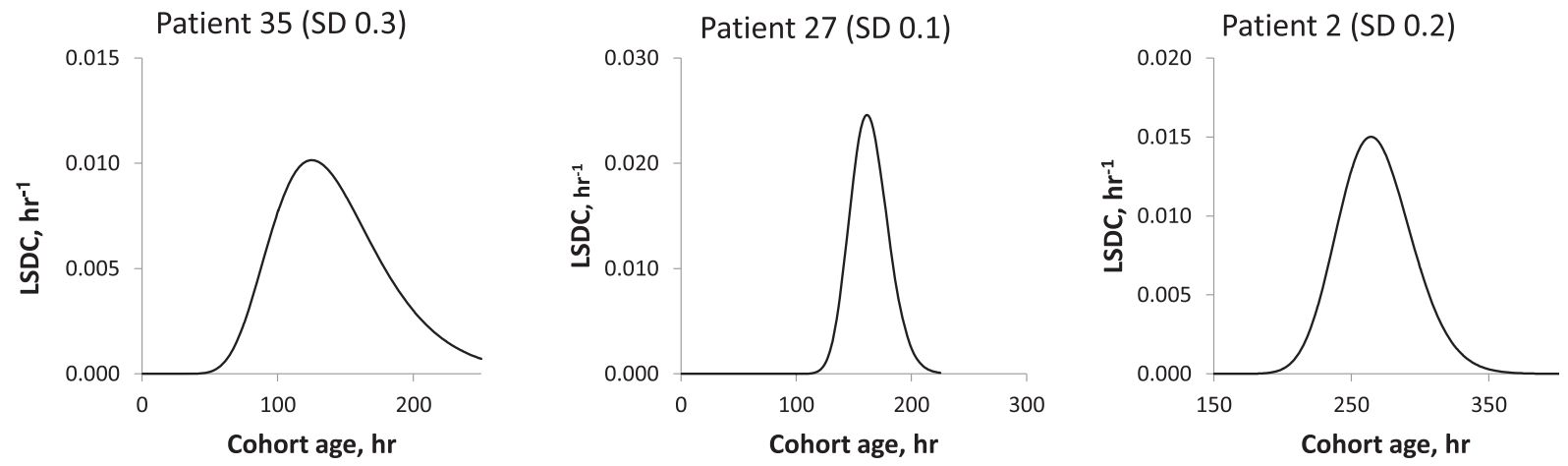

Fig. 3 Optimal parameter value searches and lifespan distributions. Top: The examples shown yielded optima in three different SD-defined parameter spaces. The low points on the SS value curves define the optimal parameter value sets. Resolution is $0.25 \% / h$ (patients 35,2$), 1 \% / h$ (patient 27). Bottom: the optimal fractional lifespan dependent consumption rate (LSDC) distributions for these optima are shown. For example, the optimal parameter values for patient 35 demonstrate the distribution of lifespan-dependent consumption rates per cohort (peaking at $1 \% / \mathrm{h}$ ) shown at left. Resolution is $0.5 \mathrm{hr}$ 


\section{A) Examples of parameter-generated optimal consumption curves}
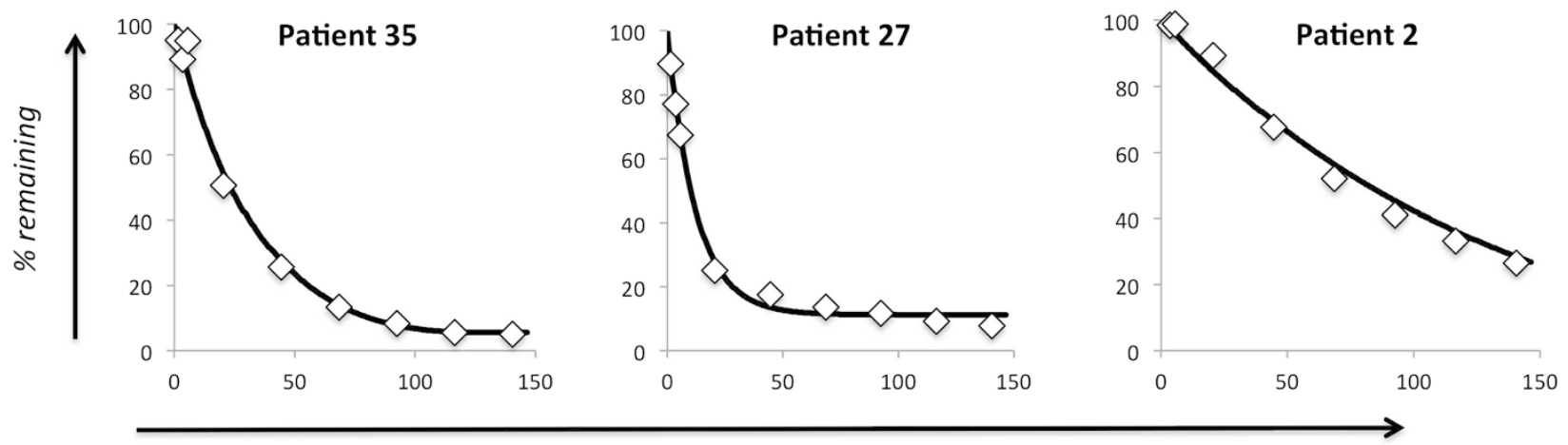

hours after platelet infusion

B) Relationship between RD value and variance of LS estimate

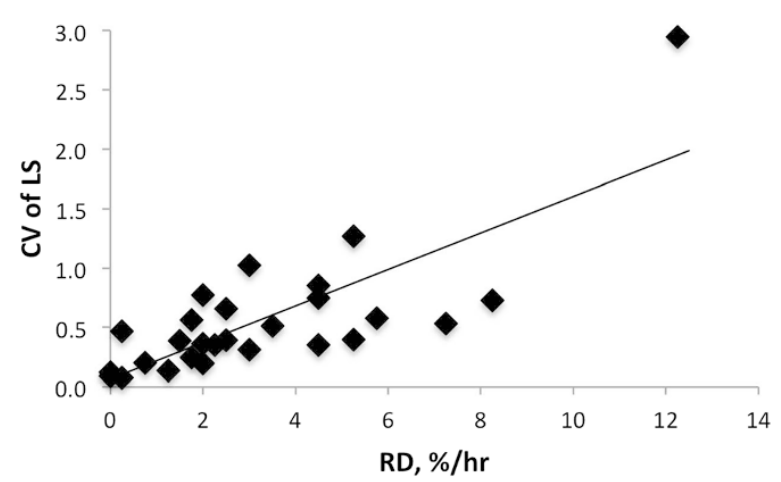

Fig. 4 Modeled consumption curves, LS variance. a Examples of parameter-generated optimal modeled consumption curves. The data points shown for each patient were used to infer optimal parameter values (RD, LS, SD, and LL; Table 1) predictive of the consumption curves shown. b Relationship between RD value and variance of LS. Values are from Table 1

used to analyze each complete data set (at the SD value of the complete data set's global minimum). We found CV values for calculated RD and LL parameters to be under 0.5 for over $75 \%$ of these cases (Table 1). Quantification of platelet lifespan was more difficult, with only $52 \%$ of our cases showing CV values for the LS parameter of under 0.5 . That is expected, however, because LS value estimates showed a larger variance in cases where random destruction predominated (Fig. 4b).

\section{Predicting the effects of reduced platelet production and increased random destruction}

As a guide to interpreting the parameter values in Table 1, we used the model to predict how a normal platelet population's consumption parameter values might shift in response to A) impaired production, B) increased consumption, or $\mathrm{C}$ ) increased consumption in association with a homeostatic increase in platelet production. Our assumptions were: i) The optimal parameter values $\left(\mathrm{RD}_{0}, \mathrm{LS}_{0}\right.$, and $\mathrm{SD}_{0}$ ) and the associated platelet production rate $\left(\mathrm{PR}_{0}\right)$ obtained for the three patients in the study whose platelet counts transiently normalized in response to prednisone (patients 3,33 , and 38 , Table 1) are representative of normal.

ii) RD is comprised of two component processes: Hemostatic RD (HRD) and non-hemostatic RD (NHRD) (i.e. RD = HRD + NHRD). Substantial hepatic NHRD is a well characterized phenomenon [18].

iii) The absolute HRD value at a normal platelet count $\left(\mathrm{aHRD} \mathrm{D}_{0}\right)$ makes up a given normal fraction (" $\mathrm{f}$ ") of absolute RD (i.e. $\mathrm{aHRD}_{0} / \mathrm{RD}_{0}=\mathrm{f}$ ). We do not know the normal value of $f$.

iv) $\mathrm{aHRD}_{0}$ is maintained, as platelet count declines, via an increase in HRD and a resultant increase in $\mathrm{RD}$, as suggested by earlier studies of platelet turnover [8]. 


\section{Patient data sets}

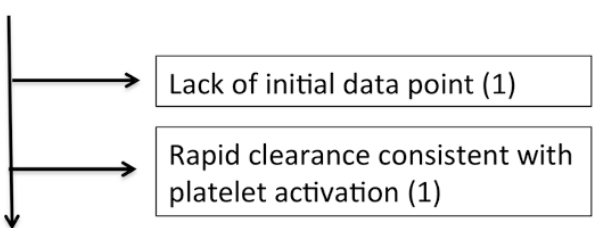

39 evaluable data sets

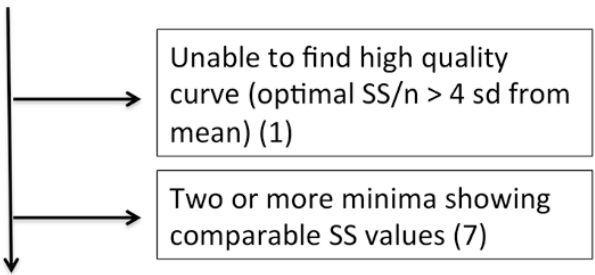

31 parameter value sets

Fig. 5 Data quality evaluation. Schematic showing criteria used to remove non-evaluable data sets from evaluation. The first data point obtained for the patient lacking an initial data point was obtained at $6 \mathrm{~h}$ after infusion of labeled platelets v) LS is not affected by reduced platelet production. Studies of the genetic basis of platelet lifespan support this assumption [3].

The effect of reduced platelet production (PR) on RD and platelet count was modeled as shown in Fig. 6. The process begins (step A) with the optimal (baseline) parameter values for pooled data from the three patients who transiently normalized their platelet counts (Table 1), using an initial " $\mathrm{f}$ " value of 1.0. From this set, a "target" reduced platelet production rate $\left(\mathrm{PR}_{1}\right)$ is generated, corresponding to $90 \%$ of $\mathrm{PR}_{0}$. Using that value, the model generates the expected (reduced) aHRD value $\left(a H R D_{1}\right)$. We then (step $B$ ) incrementally increase HRD until the model generated value of aHRD $\left(\mathrm{aHRD}_{\mathrm{i}}\right)=\mathrm{aHRD}_{0}$. The associated $\mathrm{RD}_{\mathrm{i}}$ value $\left(=\mathrm{HRD}_{\mathrm{i}}+\right.$ $\mathrm{NHRD}_{0}$ ) and platelet count values are those predicted to occur at $\mathrm{PR}_{1}$. Finally (step $\mathrm{C}$ ), we repeat steps $\mathrm{A}$ and $\mathrm{B}$ with a series of reduced platelet production rates $\left(\mathrm{PR}_{\mathrm{i}}\right)$. This generates predicted $\mathrm{HRD}$ and platelet count values for each $\mathrm{PR}_{\mathrm{i}}$ value. We then repeated this analysis at $\mathrm{f}$ values of 0.5 and 0.2 .

We note that for our baseline parameter values, $\mathrm{aRD}_{0}$ (RD $\times$ platelet count) is equal to $45 \%$ of $\mathrm{PR}_{0}$. We make no quantitative predictions for the effect of reducing $P R$

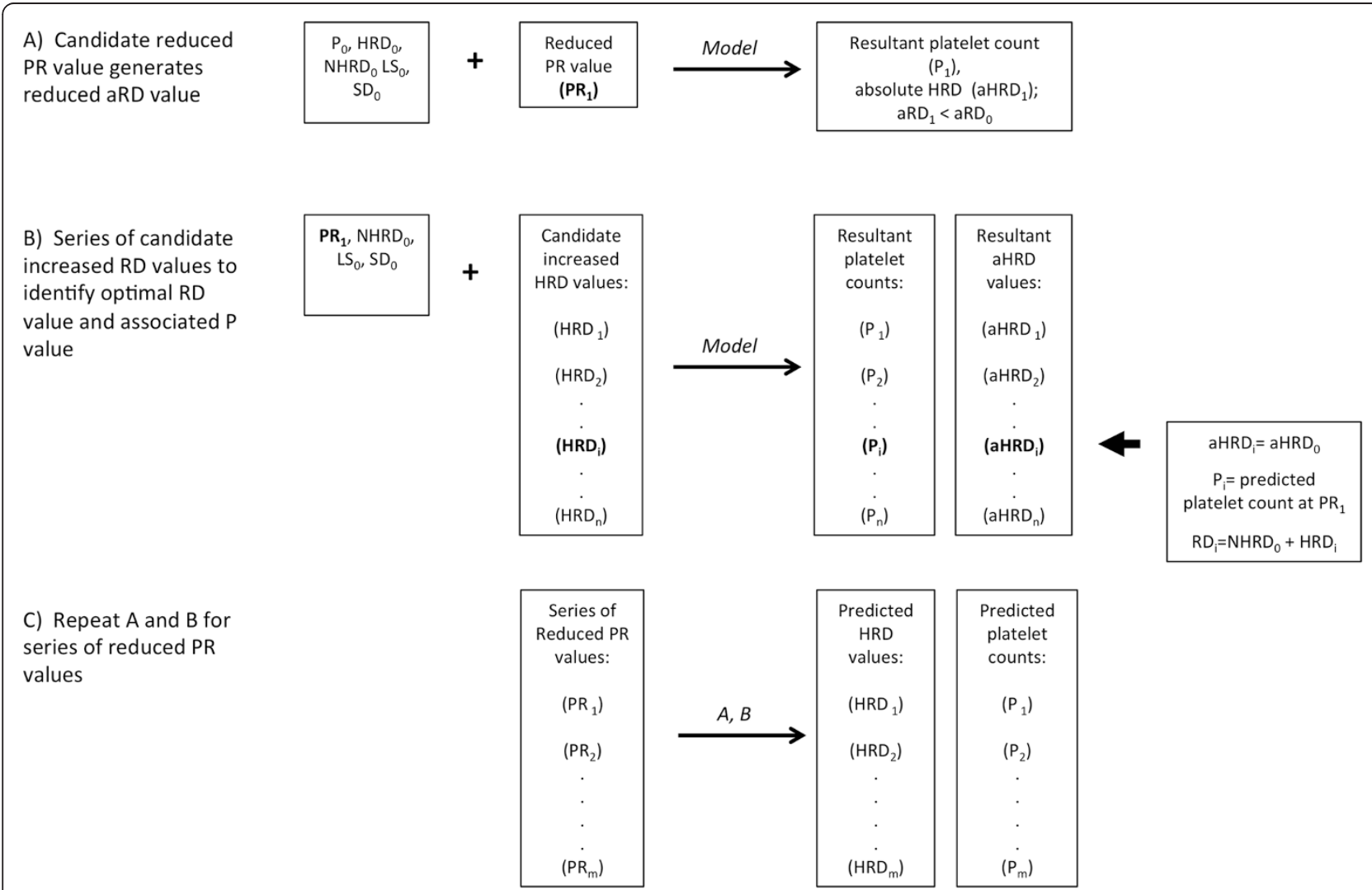

Fig. 6 Modeling the effect of reduced Production rate on HRD and Platelet count. Schematic of the interpolation process described in the text 
below $\mathrm{aRD}_{0}$ because the assumptions underlying the current numerical analysis model may not hold in that case. This is because the number of hemostatic targets is expected to increase below platelet counts at which hemostasis begins to be impaired. That in turn would invalidate the assumption of a constant absolute HRD rate, which is one of the bases for our iterative predictive method (Fig. 5). A model incorporating a dynamic hemostatic target population will be needed to predict platelet consumption rates in these circumstances.

To model the effect of increased random platelet consumption (RD), we generated a series of incrementally reduced target platelet counts $\left(\mathrm{P}_{\mathrm{i}}\right)$ (range: $90 \%$ to $10 \%$ of baseline), and to achieve each we incrementally increased RD from its baseline value until the model generated value of $\mathrm{P}\left(\mathrm{P}_{\mathrm{m}}\right)$ was equal to $\mathrm{P}_{\mathrm{i}}$. To model the concurrent effects of increased RD and homeostatically increased PR, we used the same series of target platelet counts $\left(\mathrm{P}_{\mathrm{i}}\right)$, and for each we increased $\mathrm{PR}$ in a manner proportional to the reduction in platelet count (to a maximum of twice the baseline PR value, a conservative theoretical starting point) before, again, empirically identifying $\mathrm{RD}_{\mathrm{i}}$.

The results of these three modeling approaches are plotted with the values obtained for the patients in Fig. 7.

\section{Optimal patient parameter values in comparison to modeled values}

Surprisingly, only four patients in the study showed a platelet production rate that is even modestly increased $(>50 \%)$ in comparison to the presumed normals (Fig. $7 \mathrm{c}$ ). The latter showed a mean platelet production rate $(2.12 \mathrm{~K} / \mathrm{ul} / \mathrm{h})$ comparable to the $1.7 \mathrm{~K} / \mathrm{ul} / \mathrm{h}$ rate estimated for normals in a previous study [8]. The finding of predominantly low to normal production rates in the thrombocytopenic cases (Fig. 7a) is corroborated by the distribution of random destruction rates (Fig. 7b), where

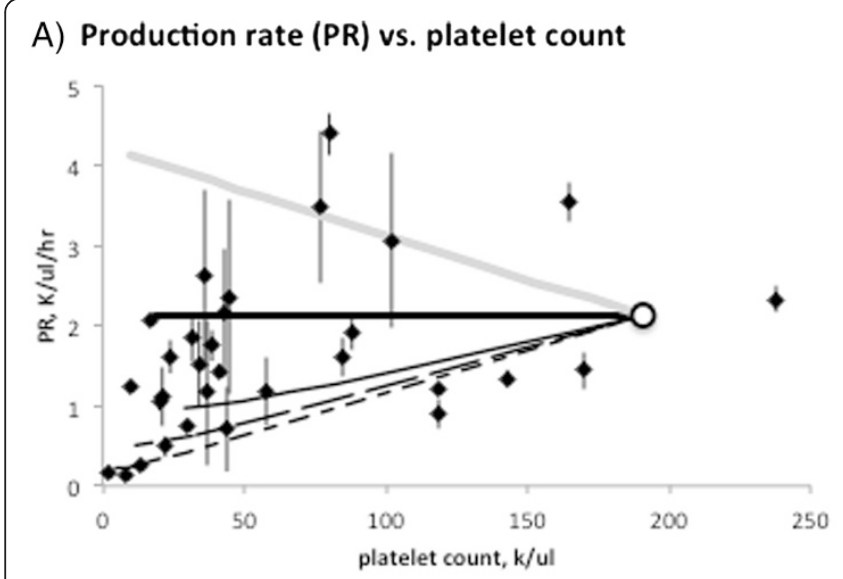

B) Random destruction rate (RD) vs. platelet count

\section{C) RD vs PR}
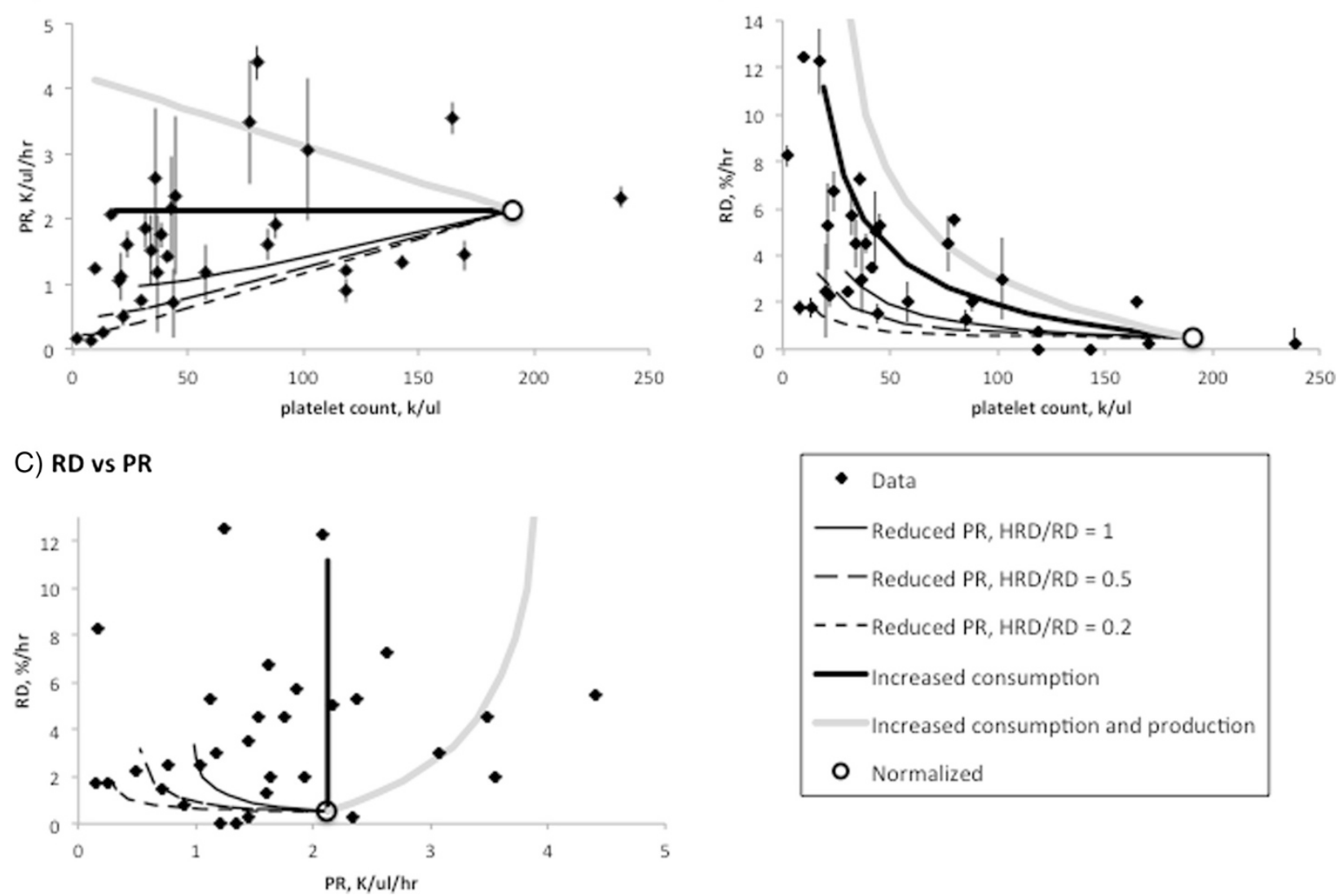

Fig. 7 Platelet production and consumption, observed and predicted parameter values. Optimal RD and PR values from Table 1 are plotted for each of the patients in the study. Projected values for thrombocytopenias due to reduced production (at three values of HRD/RD), increased consumption with no homeostatic increase in production, and increased consumption with a compensatory increase in production rate, were interpolated as described in the text. a Population turnover rate, which at equilibrium equals platelet production rate, vs. platelet count. b Random destruction rate (RD) vs. platelet count. $\mathbf{c}$ RD and PR values from $\mathbf{a}$ and $\mathbf{b}$. Error bars are standard deviations arrived at via jacknife resampling (see text) 
rates consistent with no increase in platelet production are seen for, again, all but a handful of the patients. A surprisingly large number of cases (at least 12 of 31) fall near the $\mathrm{RD}$ rates predicted to result solely from impaired platelet production. The predicted rates vary significantly, however, as a function of $a \mathrm{HRD}_{0} / \mathrm{RD}_{0}$ (' $\mathrm{f}$ ').

Because we don't know the normal value of ' $\mathrm{f}$ ', our ability to predict the increase in $\mathrm{RD}$ at low platelet counts is limited. Future studies in patients with thrombocytopenias due to impaired platelet production could resolve that problem.

\section{Modeling of immature platelet fraction values}

An ability to take up fluorescent marker dyes such as thiazole orange (a marker of "reticulated platelets", RP) or the proprietary dyes used in Sysmex hematology analyzers (marking the "immature platelet fraction", IPF) is thought to be characteristic of those platelets which have recently been released into the bloodstream. The age threshold (T) at which "young' platelets stop taking up these marker dyes is not known. Because the numerical analysis model generates a platelet age distribution for any given set of parameter values, it can be used both to estimate $\mathrm{T}$ and to predict the effect of altered production and consumption rates on the fraction of platelets of age less than T (i.e. the IPF).

Specifically, the normal range for the IPF is approximately $4.5 \%$ (each clinical laboratory typically establishes its own range; this is the value in use at the Memphis VA Medical Center). Per the age distribution predicted by the model for our normalized controls (Fig. 8a), the youngest $4.5 \%$ of platelets corresponds to those aged less than $4 \mathrm{~h}$ (i.e. $\mathrm{T}=4 \mathrm{~h}$ ). Application of that cutoff to the age distributions generated during modeling of the effects of altered production and/or consumption (Fig. 7), generates the predicted IPF and absolute IPF (aIPF) for thrombocytopenias induced by those mechanisms, as shown in Fig. 8b.

We note that this analysis depends on the assumption that all nascent platelets below a given age $(\mathrm{T})$ take up the fluorescent markers used in the RP and IPF assays. Our measurements of mass turnover for mature and reticulated murine platelets suggest that this may not be the case [19].

A) Inferred age distribution histograms
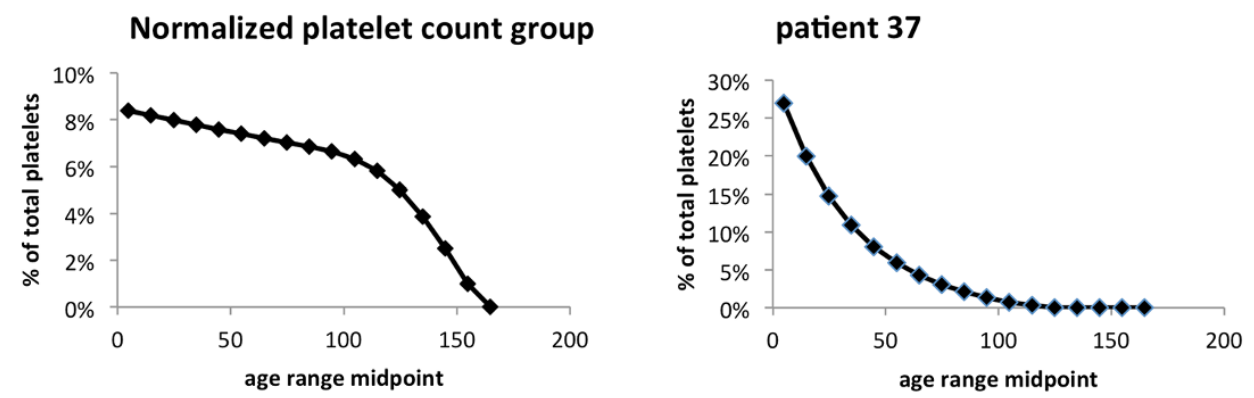

B) Modeled IPF and absolute IPF (aIPF) values
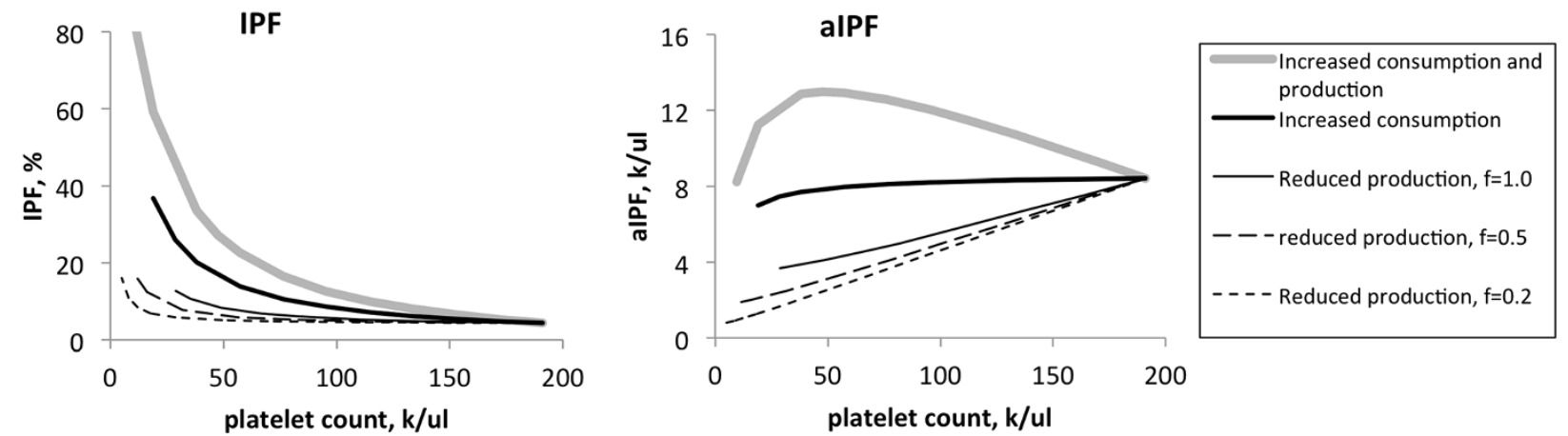

Fig. 8 Age distribution histograms and IPF modeling. a Histograms were generated from the optimal kinetic parameter values shown in Table 1 using bin sizes of $10 \mathrm{~h}$. b Projected IPF and absolute IPF (aIPF) were generated from the parameter values for the normalized platelet count group (Table 1), as described in the text 


\section{Conclusions}

Here we have shown the feasibility of quantifying platelet consumption and production rate parameters via numerical analysis of clinical autologous ${ }^{111}$ Inlabeled platelet consumption data. Although in some cases more than one set of rate parameters yields a consumption curve that closely fits the in vivo platelet consumption data, we were able to identify a single "global" optimum parameter set for $79 \%$ of the evaluable data sets. We have shown that the technical challenges associated with quantifying the variable amount of long-lived labeled cells in these studies are tractable. It would however be preferable to avoid the need for such calculations via the use of fluorescently labeled, rather than radiolabeled, platelets in this type of study.

Only a small fraction of the prednisone-refractory ITP patients in this study (4 of 39 evaluable patients) showed evidence of a compensatory increase in platelet production rate. Platelet production rates below those of the presumed normals were frequent, and for several patients a thrombocytopenia due solely to impaired platelet production could not be ruled out. More data from normal controls would be needed to confirm these conclusions, as would studies aimed at quantifying the normal (absolute) rate of random hemostatic platelet consumption. Also, it remains possible that a strong homeostatic increase in platelet production rate is characteristic of those ITP patients who demonstrate a durable response to prednisone. If they can be correlated with other types of study (such as evaluation of the immunologic effects on megakaryocyte function that others have reported [20]) [21]), our findings would be consistent with the normal serum thrombopoietin (TPO) levels seen in most ITP patients [22-25]. That observation remains difficult to explain.

Finally, our modeling of the impact of changes in platelet production and consumption rates on the platelet age distribution suggest that there is no simple correlation between the aIPF and the etiology of a given thrombocytopenia. Despite the fact that the platelet count is used to calculate the aIPF, the aIPF can only be interpreted in the context of the platelet count (Fig. 8b). But if the predicted curves in Fig. $8 \mathrm{~b}$ can be verified by comparison of IPF values to measured clinical platelet production and consumption rates in bone marrow failure patients, the IPF and platelet count could subsequently be used to infer the kinetic bases for most thrombocytopenias.

\section{Additional file}

Additional file 1: Optimal parameter search results. (DOCX $26 \mathrm{~kb}$ )

\section{Abbreviations}

RD: Random destruction (\%/h); LS: Intrinsic lifespan (hr); SD: Standard deviation of In LS; PR: Production rate (K/ul/h); AD: Age distribution; ITP: Immune thrombocytopenic purpura; CV: Coefficient of variation (stdev/ mean); IPF: Immature platelet fraction (\%); aIPF: Absolute immature platelet fraction (K/ul/h); LL: Long lived species (\%); SS: Sum of squared residuals value; SS/n: SS value normalized to the number of data points in a given data set; $\mathrm{RD}_{0}, \mathrm{LS}_{0}, \mathrm{SD}_{0}$ : Optimal parameter values for the "normalized" subset of patients; HRD: Hemostatic random destruction (\%/h); NHRD: Nonhemostatic random destruction (\%/h); aHRD, aNHRD: Absolute values of HRD and NHRD (K/ul/h); HRD, RD, Values of $\mathrm{HRD}$ and RD for the "normalized" subset of patients; "f": Ratio of aHRD 0 to $\mathrm{RD}_{0} ; \mathrm{P}_{\mathrm{i}}$ : Platelet count at rank "I" in the interpolation process (Fig. 6); RP: Reticulated platelets; T: Threshold age below which platelets are identified as "immature".

\section{Competing interests}

The numerical analysis model is disclosed in a patent application filed in the USPTO December 2012, naming the author (T.S. Strom) as inventor.

\section{Authors' contributions}

TS designed the numerical analysis model, performed the data analysis, and wrote the paper.

\section{Acknowledgements}

We thank Dr. Manel Roca (Department of Nuclear Medicine, Hospital Universitari de Bellvitge, L'Hospitalet de Llobregat, Spain) and Dr. Nuria Pujol-Moix (Platelet Pathology Unit, Hospital de la Santa Creu I Sant Pau, Universitat Autonoma de Barcelona, Spain) for sharing of clinical and laboratory data and assisting in the data analysis. Model development by TS (as previously applied to animal studies) was supported by R01Al071163 (David J. Rawlings, Principal Investigator), by NIAID 1R21Al079757-01A1 (TS), and by the Department of Veterans Affairs.

Received: 21 August 2014 Accepted: 5 October 2015

Published online: 19 October 2015

\section{References}

1. Recommended methods for radioisotope platelet survival studies: by the panel on Diagnostic Application of Radioisotopes in Hematology, International Committee for Standardization in Hematology. Blood 1977;50(6):1137-1144.

2. Lotter MG, Heyns AD, Badenhorst PN, Wessels P, Martin van Zyl J, Kotze HF, et al. Evaluation of mathematic models to assess platelet kinetics. J Nucl Med. 1986;27(7):1192-201.

3. Dowling MR, Josefsson EC, Henley KJ, Hodgkin PD, Kile BT. Platelet senescence is regulated by an internal timer, not damage inflicted by hits. Blood. 2010;116(10):1776-8.

4. Dornhorst AC. The interpretation of red cell survival curves. Blood. 1951;6(12):1284-92.

5. Mills J. The life-span of the erythrocyte. J Physiol Soc. 1946;105 (16P).

6. Murphy EA, Francis ME. The estimation of blood platelet survival. II. The multiple hit model. Thromb Diath Haemorrh. 1971;25(1):53-80.

7. Ballem PJ, Segal GM, Stratton JR, Gernsheimer T, Adamson JW, Slichter SJ. Mechanisms of thrombocytopenia in chronic autoimmune thrombocytopenic purpura. Evidence of both impaired platelet production and increased platelet clearance. J Clin Invest. 1987;80(1):33-40.

8. Hanson SR, Slichter SJ. Platelet kinetics in patients with bone marrow hypoplasia: evidence for a fixed platelet requirement. Blood. 1985;66(5):1105-9.

9. Tomer A, Hanson SR, Harker LA. Autologous platelet kinetics in patients with severe thrombocytopenia: discrimination between disorders of production and destruction. J Lab Clin Med. 1991;118(6):546-54.

10. Strom TS. A numerical analysis model for the interpretation of in vivo platelet consumption data. PLoS One. 2013;8(1), e55087.

11. Dowling MR, Josefsson EC, Henley KJ, Kile BT, Hodgkin PD. A model for studying the hemostatic consumption or destruction of platelets. PLoS One. 2013;8(3), e57783.

12. Roca M, Muniz-Diaz E, Mora J, Romero-Zayas I, Ramon O, Roig I, et al. The scintigraphic index spleen/liver at 30 minutes predicts the success of splenectomy in persistent and chronic primary immune thrombocytopenia. Am J Hematol. 2011;86(11):909-13. 
13. von dem Borne AE, Helmerhorst FM, van Leeuwen EF, Pegels HG, von Riesz E, Engelfriet CP. Autoimmune thrombocytopenia: detection of platelet autoantibodies with the suspension immunofluorescence test. $\mathrm{Br}$ J Haematol. 1980;45(2):319-27.

14. Aster RH. Pooling of platelets in the spleen: role in the pathogenesis of "hypersplenic" thrombocytopenia. J Clin Invest. 1966;45(5):645-57.

15. AuBuchon JP, Herschel L, Roger J. Further evaluation of a new standard of efficacy for stored platelets. Transfusion. 2005;45(7):1143-50.

16. Holme S, Heaton A, Roodt J. Concurrent label method with $111 \mathrm{ln}$ and $51 \mathrm{Cr}$ allows accurate evaluation of platelet viability of stored platelet concentrates. Br J Haematol. 1993;84(4):717-23.

17. Shao J, Wu CFJ. A general theory for jackknife variance estimation. The Annals of Statistics. 1989;17(3):1176-97.

18. Hoffmeister KM. The role of lectins and glycans in platelet clearance. J Thromb Haemost. 2011;9 Suppl 1:35-43.

19. Prislovsky A, Marathe B, Hosni A, Bolen AL, Nimmerjahn F, Jackson CW, et al. Rapid platelet turnover in WASP(-) mice correlates with increased ex vivo phagocytosis of opsonized WASP(-) platelets. Exp Hematol. 2008;36(5):609-23.

20. McMillan R, Wang L, Tomer A, Nichol J, Pistillo J. Suppression of in vitro megakaryocyte production by antiplatelet autoantibodies from adult patients with chronic ITP. Blood. 2004;103(4):1364-9.

21. Guo L, Yang L, Speck ER, Aslam R, Kim M, McKenzie CG, et al. Allogeneic platelet transfusions prevent murine T-cell-mediated immune thrombocytopenia. Blood. 2014;123(3):422-7.

22. Aledort LM, Hayward CP, Chen MG, Nichol JL, Bussel J. Prospective screening of 205 patients with ITP, including diagnosis, serological markers, and the relationship between platelet counts, endogenous thrombopoietin, and circulating antithrombopoietin antibodies. Am J Hematol. 2004;76(3):205-13

23. Emmons RV, Reid DM, Cohen RL, Meng G, Young NS, Dunbar CE, et al. Human thrombopoietin levels are high when thrombocytopenia is due to megakaryocyte deficiency and low when due to increased platelet destruction. Blood. 1996;87(10):4068-71.

24. Kappers-Klunne MC, de Haan M, Struijk PC, van Vliet HH. Serum thrombopoietin levels in relation to disease status in patients with immune thrombocytopenic purpura. Br J Haematol. 2001;115(4):1004-6.

25. Makar RS, Zhukov OS, Sahud MA, Kuter DJ. Thrombopoietin levels in patients with disorders of platelet production: diagnostic potential and utility in predicting response to TPO receptor agonists. Am J Hematol. 2013;88(12):1041-4.

\section{Submit your next manuscript to BioMed Central and take full advantage of:}

- Convenient online submission

- Thorough peer review

- No space constraints or color figure charges

- Immediate publication on acceptance

- Inclusion in PubMed, CAS, Scopus and Google Scholar

- Research which is freely available for redistribution 